\title{
Editorial
}

\section{Therapeutic targeting based on gene-expression profiling in vestibular schwannomas}

Pier Paolo Peruzzi, M.D., Ph.D., AND RusSELl R. LONSER, M.D.

\section{Department of Neurological Surgery, The Ohio State University Wexner Medical Center, Columbus, Ohio}

Dr. Agnihotri and colleagues analyzed the differential gene expression between vestibular schwannomas (VSs) and normal control vestibular nerve tissue. ${ }^{1}$ They uncovered a rather striking pattern of gene dysregulation in VSs. More than 1400 and 2800 genes were downregulated or upregulated, respectively, in VSs compared to vestibular nerves. Furthermore, the authors found similar gene expression patterns between sporadic and neurofibromatosis Type 2 (NF2)-associated VSs. A statistical analysis of deregulated gene expression and functional clustering into known molecular pathways revealed that several pathways are aberrant in VSs compared to vestibular nerve. Specifically, the authors found that the PIK3/ ATK/mTOR (mammalian target of rapamycin) signaling cascade was an important pathway that was overexpressed in VSs. To assess the therapeutic outcome of inhibiting this pathway, the authors examined the in vitro effect of 2 new selective PIK3/AKT/mTOR inhibitors (BEZ235 and PKI-587) on VS cells and found that treatment reduced cellular proliferation and increased apoptosis.

Based on tumor size, patient age, hearing status, and/ or association with NF2, VSs have been managed conservatively, by resection, and/or by the use of stereotactic/ fractionated radiation..$^{2,3,8}$ Nevertheless, VSs that require treatment can be difficult to eradicate using available therapies, and treatment can be associated with neurological complications related to VS proximity to cranial nerves/vital structures, tumor size, and/or the multicentricity of tumors in syndromic cases. The work by Dr. Agnihotri and colleagues provides deeper insights into the molecular biology of VSs and the basis for new, targeted therapeutic approaches to these tumors. Specifically, the authors found that the most significant upregulated signaling pathway in VSs was the PI3K/AKT/mTOR cascade, which is also upregulated in glioblastoma multiforme and medulloblastoma. ${ }^{9,14}$ Exploiting these findings, the authors successfully treated VS cells in vitro with new putative therapeutic compounds (BEZ235, PKI-587).

The authors found that there was not a substantial gene expression difference between sporadic and familial
(NF2-related) VSs. This is in contradistinction to many central nervous system tumors, including glioblastoma multiforme and medulloblastoma. ${ }^{7,10-12}$ Prior studies have shown that these tumors display heterogeneous molecular signatures, findings that have led to the development of tumor "subgroups." These molecularly based subgroups have clinically significant behavior patterns and prognostic implications. ${ }^{710-12}$ Alternatively, the similar transcriptional signature found in sporadic and familial VSs by Dr. Agnihotri and colleagues is important for several reasons. The similar expression patterns underscore the potential critical role of the tumor suppressor NF2 gene as a fundamental aberration in VSs., ${ }^{3,6}$ This directs further efforts in dissecting the role of the NF2 gene as a controller of the PIK3/AKT/mTOR pathway. Finally, it suggests that common pathways can be successfully targeted across the VSs (sporadic and NF2-associated) and have similar therapeutic effects.

Consistent with the current findings, PIK3, AKT, and/ or mTOR upregulation in VSs had been shown both by gene expression analysis and immunohistochemistry., ${ }^{4,513}$ Others have described the role of merlin (the protein produced by the NF2 gene) as a direct negative regulator of mTOR. ${ }^{6}$ Because of the involvement of these pathways in cell cycle activation/apoptosis inhibition, they have been found frequently overexpressed in cancers and represent a therapeutic target for inhibition leading to growth arrest and apoptosis. More important, and pragmatic as well, is the approach of targeting several points in this molecular pathway, as employed by the authors in assessing biological efficacy in cultured VS cells. Using compounds that are able to potently target upregulated portions of a critical pathway (or putative therapeutic compounds that can target multiple critical oncologic pathways) represents an important mechanistic approach to chemotherapeutic treatment of these tumors, as the authors have shown in cell culture experiments.

Additional future studies will be valuable to enhance understanding of the current findings and the development of biological agents for VS treatment. Further validation of genomic findings could be helpful. While VS specimens were collected fresh from living patients, control vestibular nerve cells were necessarily obtained from cadavers. Thus, changes in $\mathrm{pH}$, temperature, and oxygen tension could have altered mRNA stability and array output. Microarray findings could be further validated at the cellular level, either by comparing mRNA from VS cells to mRNA from normal Schwann cells or by comparing the differential mRNA expression before and after silencing of NF2 in cultured Schwann cells. The authors could also show that the therapeutic compounds they used, BEZ235 and PKI- 
587, are superior to other available inhibitors of pathways involved in VS oncogenesis. Finally, future in vivo studies in animal models could be useful to demonstrate that drugs reach therapeutic levels within VSs after systemic delivery and provide a therapeutic response.

While additional work will be needed to attain a more complete understanding of VS biology that leads to new clinical treatments, the findings of the current study represent a critical step forward in understanding VS and developing targeted therapies. The authors should be commended for this important work. This study is further evidence that comparative gene-expression analysis has the potential to uncover information that will allow for a better understanding of tumor biology and tumorigenic pathway interactions. These findings will permit the formulation of new, targeted therapeutic strategies for treatment of VS. We look forward to further studies by this group and others (based on this work) into VS.

(http://thejns.org/doi/abs/10.3171/2014.2.JNS14321)

\section{Disclosure}

The authors report no conflict of interest.

\section{References}

1. Agnihotri S, Gugel I, Remke M, Bornemann A, Pantazis G, Mack SC, et al: Gene-expression profiling elucidates molecular signaling networks that can be therapeutically targeted in vestibular schwannoma. Laboratory investigation. J Neurosurg [epub ahead of print Month Day, 2014. DOI: 10.3171/2014.6.JNS131433]

2. Al-Shudifat AR, Kahlon B, Hoglund P, Soliman AY, Lindskog K, Siesjo P: Age, gender and tumour size predict work capacity after surgical treatment of vestibular schwannomas. J Neurol Neurosurg Psychiatry 85:106-111, 2014

3. Asthagiri AR, Parry DM, Butman JA, Kim HJ, Tsilou ET, Zhuang Z, et al: Neurofibromatosis type 2. Lancet 373:19741986, 2009

4. Fong B, Barkhoudarian G, Pezeshkian P, Parsa AT, Gopen Q, Yang I: The molecular biology and novel treatments of vestibular schwannomas. A review. J Neurosurg 115:906-914, 2011

5. Jacob A, Lee TX, Neff BA, Miller S, Welling B, Chang LS: Phosphatidylinositol 3-kinase/AKT pathway activation in human vestibular schwannoma. Otol Neurotol 29:58-68, 2008

6. James MF, Han S, Polizzano C, Plotkin SR, Manning BD, Stemmer-Rachamimov AO, et al: NF2/merlin is a novel negative regulator of mTOR complex 1 , and activation of mTORC1 is associated with meningioma and schwannoma growth. Mol Cell Biol 29:4250-4261, 2009

7. Liang Y, Diehn M, Watson N, Bollen AW, Aldape KD, Nicholas MK, et al: Gene expression profiling reveals molecularly and clinically distinct subtypes of glioblastoma multiforme. Proc Natl Acad Sci U S A 102:5814-5819, 2005

8. Milligan BD, Pollock BE, Foote RL, Link MJ: Long-term tumor control and cranial nerve outcomes following Gamma Knife surgery for larger-volume vestibular schwannomas. Clinical article. J Neurosurg 116:598-604, 2012

9. Mohan AL, Friedman MD, Ormond DR, Tobias M, Murali R, Jhanwar-Uniyal M: PI3K/mTOR signaling pathways in medulloblastoma. Anticancer Res 32:3141-3146, 2012

10. Northcott PA, Korshunov A, Witt H, Hielscher T, Eberhart CG, Mack S, et al: Medulloblastoma comprises four distinct molecular variants. J Clin Oncol 29:1408-1414, 2011

11. Northcott PA, Shih DJ, Peacock J, Garzia L, Morrissy AS,
Zichner T, et al: Subgroup-specific structural variation across 1,000 medulloblastoma genomes. Nature 488:49-56, 2012

12. Noushmehr H, Weisenberger DJ, Diefes K, Phillips HS, Pujara K, Berman BP, et al: Identification of a $\mathrm{CpG}$ island methylator phenotype that defines a distinct subgroup of glioma. Cancer Cell 17:510-522, 2010

13. Rong R, Tang X, Gutmann DH, Ye K: Neurofibromatosis 2 (NF2) tumor suppressor merlin inhibits phosphatidylinositol 3-kinase through binding to PIKE-L. Proc Natl Acad Sci U S A 101:18200-18205, 2004

14. Sunayama J, Matsuda K, Sato A, Tachibana K, Suzuki K, Narita Y, et al: Crosstalk between the PI3K/mTOR and MEK/ ERK pathways involved in the maintenance of self-renewal and tumorigenicity of glioblastoma stem-like cells. Stem Cells 28:1930-1939, 2010

\section{Response}

Gelareh Zadeh, M.D., Ph.D. ${ }^{7}$ Sameer Agnihotri, Ph.D., ${ }^{1}$ Isabel Gugel, M.D., ${ }^{2}$ Marc Remke, M.D., ${ }^{1,3,4}$ Antje Bornemann, M.D., Ph.D., ${ }^{5}$ Georgios Pantazis, M.D., 6 Stephen C. Mack, B.Sc., ${ }^{1,3,4}$ David SHIH, M.Sc., ${ }^{1,3,4}$ Sanjay K. Singh, Ph.D., ${ }^{1}$ Nesrin Sabha, B.S., ${ }^{1}$ Michael D. Taylor, M.D., Ph.D., 1,3,4 Marcos Tatagiba, M.D., Ph.D., 2 and Boris KrischeK, M.D., PH.D. ${ }^{2,8}$

\begin{abstract}
${ }^{1}$ Arthur \& Sonia Labatt Brain Tumour Research Centre and ${ }^{3}$ Program in Developmental \& Stem Cell Biology, The Hospital for Sick Children, Toronto; ${ }^{4}$ Department of Laboratory Medicine \& Pathobiology, University of Toronto; ' Division of Neurosurgery, Toronto Western Hospital, University of Toronto, Ontario, Canada; Departments of ${ }^{2}$ Neurosurgery and ${ }^{5}$ Neuropathology, University of Tübingen; ${ }^{6}$ Department of Neuropathology, University of Marburg; and ${ }^{8}$ Department of Neurosurgery, University of Cologne, Germany
\end{abstract}

We are grateful to Drs. Peruzzi and Lonser for their positive review of our work and for highlighting some of the key points arising from the results we obtained in our study. We hope our research will raise the notion that the PI3K/mTOR/AKT pathway is an attractive therapeutic target in VS. Furthermore, BEZ235 is under clinical trial for several cancers and has shown robust results in preclinical models and preliminary clinical trials (http:// www.clinicaltrials.gov). We agree with the points Drs. Peruzzi and Lonser made regarding some of the limitations of our study, including the observation that obtaining control specimens at autopsy may have resulted in technical issues when they were compared to specimens obtained surgically. In addition, we concur with the next steps they propose, including comparison of schwannoma cells with normal Schwann cells and examining the role of NF2 loss in Schwann cells to explore NF2-regulated genes in schwannoma. As they suggest, animal models could pave the way for preclinical testing to identify therapeutic strategies for tumors not amenable to resection. Further studies examining the genomics of these tumors, using gene expression or other modalities such as microRNA, and epigenetic profiling may shed some light on the molecular pathogenesis of these tumors and could lead to new therapeutic insights for VS.

Please include this information when citing this paper: published online September 23, 2014; DOI: 10.3171/2014.2.JNS14321. 